\title{
Germanica
}

\section{Années nazies - années d'après-guerre : le prisme de la mille-et-unième année dans les regards autobiographiques}

Nazizeit - Nachkriegszeit: Das Prisma des tausendhundersten Jahres

\section{Kerstin Adam}

\section{OpenEdition}

Journals

Édition électronique

URL : http://journals.openedition.org/germanica/1925

DOI : 10.4000/germanica. 1925

ISSN : 2107-0784

Éditeur

Université de Lille

\section{Édition imprimée}

Date de publication : 30 juin 1997

Pagination : 111-124

ISBN : 9782098426320

ISSN : 0984-2632

\section{Référence électronique}

Kerstin Adam, «Années nazies - années d'après-guerre : le prisme de la mille-et-unième année dans les regards autobiographiques », Germanica [En ligne], 20 | 1997, mis en ligne le 16 juillet 2013, consulté le 06 octobre 2020. URL : http://journals.openedition.org/germanica/1925 ; DOI : https:// doi.org/10.4000/germanica.1925

Ce document a été généré automatiquement le 6 octobre 2020.

(c) Tous droits réservés 


\title{
Années nazies - années d'après- guerre : le prisme de la mille-et- unième année dans les regards autobiographiques
}

\author{
Nazizeit - Nachkriegszeit: Das Prisma des tausendhundersten Jahres
}

\section{Kerstin Adam}

1 La période du Troisième Reich a à maintes reprises constitué une préoccupation majeure dans les écrits autobiographiques d'auteurs allemands contemporains. C'est le cas pour les textes des cinq écrivains abordés dans cette analyse, c'est-à-dire ceux de Dieter Wellershoff (Die Arbeit des Lebens ${ }^{1}$; Der Ernstfall ${ }^{2}$ ), Günter de Bruyn (Zwischenbilanz $z^{3}$ ), Max von der Grün (Wie war das eigentlich ?4), Ludwig Harig (Weh dem, der aus der Reihe tanzt ${ }^{5}$ ) et Peter Rühmkorf (Die Jahre, die Ihr kennt ${ }^{6}$ ).

Nous voulons ici nous interroger sur la façon dont les années de la période nazie et de l'après-guerre ont modelé de manière déterminante la substance des œuvres étudiées et l'approche autobiographique de ces cinq auteurs. Les critères selon lesquels nous avons sélectionné ces textes se résument d'abord au fait que les auteurs sont tous nés dans la deuxième moitié des années vingt et ont donc tous vécu les périodes en question à un stade comparable de leur évolution individuelle. Il existe certes une disparité entre eux en ce qui concerne leurs origines sociales: non seulement leurs parents appartenaient à des milieux socio-culturels différents, mais on peut aussi déceler des divergences dans l'attitude que ces parents ont eu envers le nazisme. Celleci pouvait aller d'une complicité approbatrice (dans les familles de Harig ou de Wellershoff) jusqu'à une résistance active (de la part du père de Max von der Grün) en passant par de l'indifférence plus ou moins volontaire. A notre avis, ces différences ne rendent cependant pas impossible une comparaison entre les cinq histoires individuelles, compte tenu du caractère fort nivelant du contexte dans lequel les auteurs se sont vu impliqués pendant leur jeunesse. 
3 Pour l'après 1945, on peut souligner que le devenir d'écrivain des uns et des autres ne se dessinait pas comme une carrière toute tracée. Tous furent obligés d'exercer des années durant des métiers qui ne leur laissaient que peu de temps, voire pas de temps du tout, pour se consacrer à leur production littéraire. Nous évoquons ce point commun surtout pour mieux expliquer le laps de temps relativement long qu'il y a chez ces écrivains entre l'époque illustrée et le moment où ils se penchent sur leurs jeunes années dans leurs écrits autobiographiques ; la nature de ce délai est ambivalente. C'est celle d'un détour résultant à la fois de contraintes matérielles et donc extérieures ainsi que de dispositions individuelles sur lesquelles nous allons nous interroger plus longuement.

Dans l'introduction à Die Arbeit des Lebens, Dieter Wellershoff explique que ce livre ne mérite pas la classification «autobiographie» parce qu'il est «beaucoup trop fragmentaire $»^{7}$. Si l'auteur veut par là renvoyer au caractère compilatoire de l'ouvrage, on ne peut pas lui donner tort : les textes qui le composent n'ont ni vu le jour la même année (ils datent en effet d'entre 1978 et 1984 et avaient déjà été publiés partiellement à d'autres occasions) ni n'ont été écrits avec des motivations comparables. Si, en revanche, Wellershoff veut dire par "fragmentaire» qu'une autobiographie, pour mériter cette désignation, devrait comprendre l'évocation la plus homogène et continue possible d'une période la plus longue possible, il faudrait lui reprocher d'avancer ici une ébauche de définition quelque peu naïve, dans la mesure où elle ne tient pas compte des réponses qui ont été données aux interrogations sur les possibilités de l'écriture autobiographique. On peut s'accorder sur le constat de l'impossibilité à embrasser toute une vie par le récit que l'on en fait. Il n'est en effet aucun auteur d'autobiographie, établi ou non comme écrivain, qui ait réussi la performance d'exposer au lecteur «sa vie». C'est d'autant moins le cas depuis que le moi - aussi bien le moi en tant que tel que le moi individuel ${ }^{8}$ - paraît se dérober de plus en plus et devenir, par conséquent, de plus en plus incertain comme instance narrative. Que cet état de chose ait influencé non seulement la réflexion théorique mais aussi la production de textes autobiographiques est évident. La fragmentarité de tels textes ne peut donc désormais plus passer pour critère esthétique et encore moins décider de ce qu'un récit soit admis parmi les autobiographies: elle devrait plutôt constituer une catégorie qui ouvre sur d'autres interrogations.

5 Quels sont donc les fragments qui composent Die Arbeit des Lebens? Des souvenirs d'enfance allant jusqu'au milieu des années trente, présentés sous forme de scènes d'une demi-page ; une contribution à l'anthologie Meine Schulzeit im III. Reich (éditée par Marcel Reich-Ranicki), qui porte le titre «Ein Allmachtstraum und sein Ende». Ici, Wellershoff, né en 1925, fils ainé d'un architecte-urbaniste dans une petite ville de Rhénanie, relate avec des accents psychanalytiques sa jeunesse sous le régime nazi. Evidemment incapable de comprendre que les schémas d'identification qu'on lui proposait n'étaient que la partie d'un système supérieur devenant visible pour le jeune garçon qu'il était, Wellershoff dit a posteriori avoir glissé imperceptiblement dans l'époque nazie'. Il décrit ensuite la guerre comme une orgie du narcissisme collectif et met en parallèle ses propres fantasmes mégalomanes et cette même manie qui s'était emparée de la majeure partie des Allemands ${ }^{10}$. 
6 En mai 1943, lorsque Wellershoff a dix-sept ans et demi, pour lui les choses basculent abruptement. D'un côté, et c'est là que sa personne apparaît comme témoin passif des événements historiques, il se rend compte, en écoutant les informations sur Radio Londres qui parlent de la défaite de 1'Afrikakorps, que la guerre va être perdue par les Allemands. D'un autre côté - ici Wellershoff s'attribue le rôle d'acteur sur la scène de la vie privée - il comprend qu'il lui est possible de se défaire de l'emprise de sa mère souffreteuse et possessive. C'est l'accent que Wellershoff met sur la brutalité avec laquelle il se rend compte des leçons de ces deux épisodes, qui se suivent à quelques instants d'intervalle, qui permet de voir dans ces scènes une illustration de points importants de la narratologie anthropologique que Wellershoff a développée sous différents angles dans ses écrits théoriques et a appliqué à ses romans et récits : quelle est la réaction de l'individu à un choc déclenché par une prise de conscience soudaine? Est-il capable de renoncer aux constructions auxiliaires qui se sont tout à coup avérées être illusoires et donc caduques?

7 Apparemment, Wellershoff n'a pas tiré immédiatement les conclusions de sa connaissance nouvelle, car il s'engage d'abord, pour la fin de la guerre, comme volontaire dans l'armée. Cette période est relatée aussi bien dans le chapitre suivant que, de manière beaucoup plus détaillée, dans un de ses autres livres autobiographiques, Der Ernstfall.

8 Wellershoff explique ${ }^{11}$ que cette décision de s'engager s'imposait à lui comme offrant une échappatoire. Elle était motivée par la quête d'une nouvelle identité qui lui permette plus facilement de se détacher d'une existence que Wellershoff et les jeunes gens de son âge commençaient à sentir s'effriter. Il est d'ailleurs frappant de voir qu'ici, comme dans les textes sur les deux années qui vont suivre, l'auteur emploie fréquemment le pluriel, surtout dans les passages de réflexion. Il ne s'agit pas de lui reprocher d'éventuelles généralisations mais plutôt de souligner que le 8 mai 1945 constitue avant tout un point tournant dans le récit de Wellershoff non pas parce que sa vie quotidienne aurait changé significativement du jour au lendemain, mais parce qu'il marque pour ainsi dire la fin du pluriel, le moment où selon l'auteur «le drame collectif éclata en des millions et des millions de drames individuels $»^{12}$.

9 En admettant la pertinence de l'esquisse définissant les critères auxquels une autobiographie doit correspondre selon Wellershoff, force est de constater que l'œuvre de Günter de Bruyn s'en rapproche plus que la sienne. L'auteur berlinois est né dans un milieu proche de la petite bourgeoisie commerçante. Sa famille est empreinte d'un catholicisme, omniprésent sans être orthodoxe, qui lui permet de se définir par rapport au national-socialisme. De Bruyn présente avec Zwischenbilanz le texte d'un inventaire certes provisoire jusqu'à dans son titre, mais chronologique et homogénéisé par l'obéissance à la propre esthétique de cet auteur.

10 A la différence de celles de Wellershoff, les réflexions théoriques de Günter de Bruyn qui trouvent ici leur illustration ne portent pas sur la production littéraire en général mais sur l'écriture autobiographique en particulier. On les trouve exposées dans Das erzählte Ich - Über Wahrheit und Dichtung in der Autobiographie ${ }^{13}$. La règle suprême de l'auteur autobiographique ne doit selon de Bruyn pas être la recherche de l'authenticité la plus totale de ce qu'il présente au public. Ayant laissé derrière lui la quête de la 
vérité autobiographique comme illusoire dans son objectif et irréaliste dans son ambition, bref comme dépassée en tant que maxime pour l'auteur moderne, Günter de Bruyn s'efforce d'exposer une vérité autobiographique sans jamais taire qu'il s'agit en fait d'une des vérités possibles. La difficulté pour l'écrivain réside à la fois dans le choix à effectuer en permanence et dans la manière avec laquelle on confère à l'événement que l'on a sélectionné pour l'intégrer dans le récit ce par quoi sa présence s'y justifie véritablement, c'est-à-dire une littérarité. Par cette littérarité autobiographique, Günter de Bruyn entend la mise en valeur du lien - artificiellement établi - entre le vécu personnel et le rôle attribué au contexte extérieur dans lequel il s'est produit, rôle qu'il faut faire transparaître plus ou moins explicitement. L'épisode suivant exemplifie les critères de composition de Günter de Bruyn :

Le jour de ma naissance illustre par deux événements la tendance à la catastrophe qui se dessine : Goebbels est nommé Gauleiter du NSDAP de Berlin, et la Reichsbahn et la Reichspost introduisent à minuit l'horaire de 24 heures. Ce n'est que rétrospectivement que l'on peut établir un lien entre ces deux nouveautés : la folie et la précision gagnent simultanément du terrain; tandis que l'éthique est en déconfiture, la technologie est de plus en plus raffinée; la modernisation qui ne signifie pas une césure pour l'année 1933, rendra possible le perfectionnement des tueries d'Auschwitz, de Coventry, et du front. ${ }^{14}$

11 Notons également qu'à cet égard, le respect d'une véracité historique absolue est secondaire selon de Bruyn lorsqu'il est abandonné au profit de la création de littérarité. C'est ainsi qu'il ajoute dans une subordonnée anodine qu'il n'y avait peut-être pas de coïncidence entre le jour de sa première sortie au cinéma ${ }^{15}$, à tel point impressionante pour l'enfant de six ans qu'elle en devient presque traumatisante, et celui de l'avènement d'Hitler au pouvoir, mais qu'il a fait coïncider les deux faits, comme il le dit dans Das erzählte Ich, parce que « ça collait bien ${ }^{16}$.

En s'appuyant sur ses journaux et sa correspondance de l'époque ainsi que sur des documents de la chronique familiale, de Bruyn commence par reconstituer l'histoire de sa famille et ensuite la période du nazisme sous laquelle il vit d'abord une enfance relativement paisible. Tardivement inscrit aux Jeunesses Hitlériennes, il doit servir à partir de 1943 comme auxiliaire de la DCA pendant un an pour ensuite être mobilisé d'office à l'âge de dix-sept ans. Blessé à la tête dans son unique combat où il rencontre les Russes, il est transporté dans un hôpital militaire sur le territoire tchèque. Faut-il voir une autre coïncidence factice dans le fait qu'il y voit la fin de la deuxième Guerre Mondiale le jour même de son opération? Elle se déroule dans des conditions extrêmement précaires et entraîne une amnésie passagère. On oblige de Bruyn à partir vers la capitale du Reich déchu dès le lendemain - un périple qui lui prendra trois mois et pendant lequel il recouvre peu à peu la parole, l'écriture et la mémoire.

13 La qualification double de " roman d'apprentissage » et de " panorama d'une époque »" qu'un critique a attribué à Zwischenbilanz pourrait s'appliquer au même titre à Wie war das eigentlich? de Max von der Grün, à ceci près qu'ici le principe de composition est tout autre. Précisons en premier lieu qu'il ne correspond aucunement à une poétologie propre à l'auteur, bien au contraire. C'est pour l'occasion que von der Grün a opté pour un style volontairement simple et dépouillé afin de rendre compte des dix-neuf premières années de sa vie. Ainsi, son livre se présente de façon facilement accessible 
au grand public. Il faut donner d'autant plus raison au commentateur qui en recommande la lecture comme manuel d'histoire ${ }^{18}$ que Max von der Grün fait alterner dans chacun des 19 chapitres organisés par année l'évocation de son vécu et de sa vision personnelle des choses avec des documents officiels sur l'époque élucidée. Il est vrai que l'auteur produit par là un ouvrage autobiographique proche du documentaire, mais c'est justement par la juxtaposition que nous venons d'évoquer que l'auteur parvient à montrer l'évolution de son individu dans un contexte déterminé par des facteurs qui lui échappent mais auxquels il échappe aussi lui-même par moment. Créer après-coup des liens entre l'histoire personnelle et nationale en se positionnant sur un méta-niveau comme Günter de Bruyn l'a fait est une manière de procéder, mais c'est aussi une façon d'évoquer les événements sous une lumière harmonisatrice (quelle que soit par ailleurs la cruauté par laquelle ils se distinguent). A côté de cela, l'écriture autobiographique peut aussi acquérir une dimension littéraire lorsque l'on laisse volontairement apparaître et subsister les disparités entre le devenir individuel et collectif. Cette dimension est donnée aux événements relatés en rendant successivement lisibles les simultanéités dont une vie se compose et qui resteraient autrement dans ce que Bloch a appelé l'obscurité du moment vécu.

Le seul des cinq textes qui porte la spécification «roman » est celui de Ludwig Harig Weh dem, der aus der Reihe tanzt. C'est en effet ici que le lecteur trouve le plus d'éléments ou de critères narratifs romanesques si l'on admet que, dans l'autobiographie, l'auteur a tendance à expliciter le fait que la position qu'il prend comme narrateur n'est pas identique à celle de l'enfant qu'il était. Ludwig Harig essaie de retracer sa vie à Sulzbach dans la Sarre où il est né en 1927 dans une famille d'artisans, en se replaçant dans la perspective du garçon qui évolue dans un milieu marqué par une manifeste étroitesse d'esprit, une méfiance envers tout et tous ceux qui sont différents et dont le goût pour une assurance quelque peu sentencieuse crée un terrain propice pour que la sympathie pour le régime nazi puisse prendre racine. Harig décrit comment les rêves d'aventures de Ludwig, qui étaient pour lui une possibilité d'échapper au contexte familial et villageois étouffant, se virent réalisés lorsqu'il rejoignit les Jeunesses Hitlériennes. Le processus qui lui permet de comprendre de quelle réalité la sienne avait été une minuscule facette semble se dérouler rapidement et sans provoquer de traumatisme; il ressemble en cela à un réveil après un cauchemar - peu après la capitulation, le jeune de dix-huit ans se défait de ses croyances en jetant, apparemment plus que symboliquement, son écusson nazi ${ }^{19}$. Il est surprenant de voir que dans le récit de Harig, on ne trouve pas une seule phrase qui parle de l'époque de la fin 1945 à la fin 1949. L'auteur ayant jugé indigne de mentionner si d'autres Allemands s'étaient eux aussi débarrassés de leurs écussons et le cas échéant comment, l'autobiographie reprend seulement au moment où l'auteur arrive à Lyon, ville dans laquelle il va travailler pendant un an comme assistant d'allemand.

Les passages de réflexion dans ce dernier chapitre du livre sont occasionnés par trois types d'événements: d'une part, les contacts avec les Lyonnais, en partie d'anciens résistants, d'autre part un voyage à l'endroit où s'était trouvé son camp d'entraînement militaire et où il ne rencontre qu'une amnésie ostensible ${ }^{20}$ et finalement un deuxième séjour à Lyon datant de la fin des années 80 : celui-ci met les tentatives de se 
remémorer l'époque d'avant quarante-cinq et celle de 1949/50 sur le même plan, car la réflexion culmine toujours dans les mêmes constats du type: "Hier, c'était hier; aujourd'hui, c'est aujourd'hui » ou encore « Je ne peux pas effacer ce qui s'est passé ${ }^{21}$.

Ce qui apparaît sûrement le moins clairement dans Weh dem, der aus der Reihe tanzt, c'est-à-dire l'aspect de l'autobiographie qui fait voir en elle une confession, un compte rendu, semble néanmoins un trait commun aux quatre textes. Même si les approches individuelles des différents auteurs divergent, on peut toutefois retenir que tout un chacun a réussi à faire passer en revue avec un souci de véracité autobiographique, donc relative, la période de sa vie qu'il a passé sous le nazisme. Chaque auteur, Harig aussi, a, pour mieux comprendre son identité actuelle, éclairé une de ses identités antérieures, et ce sans passer par un ou plusieurs personnages désignés comme fictifs, mais en disant, même si c'est parfois avec incrédulité : c'était moi.

L'enfance et la jeunesse sous le Troisième Reich semblent être restés pendant longtemps le moment le plus sombre de l'évolution de l'individualité de ces auteurs en ce sens que c'est l'époque où l'individualisation était à la fois déterminée par des contraintes sociales très pesantes et celle qui n'a en même temps jamais été éclairée de manière suffisante pour rendre superflues des interrogations que les auteurs ont souhaité exposer par la voie de l'écriture autobiographique. Ce sur quoi nous allons de notre côté nous interroger maintenant appartient sans doute au domaine spéculatif, car c'est d'abord d'une absence - comparative, certes - qu'il faut faire le constat. Pourquoi le retour aux années 33 à 45 occupe-t-il tellement plus de place que n'importe quelle autre période de la vie de ces écrivains? Avant d'apporter des éléments de réponse, faisons le point sur la façon dont l'après-guerre est reflété chez les uns et les autres.

18 Comme nous l'avons vu plus haut, Ludwig Harig met entre parenthèses les quatre premières années de l'après-guerre pour parler ensuite d'une année qu'il a passée en France. Ce séjour est relaté dans la seule perspective de la personne privée qui était assurément la représentante d'un pays ennemi peu de temps auparavant, mais qui ne reflète nulle part une implication de son individu dans un contexte politique.

Chez Max von der Grün, la phrase qu'il entend au moment où il se fait arrêter par un G.I. en août 1944, alors qu'il est stationné dans l'Ouest de la France, a une valeur emblématique: "The war is over for you $»^{22}$. Sa guerre est finie, et donc également l'histoire qu'il voulait raconter. Comme par une postface, on apprend qu'à peine quinze jours plus tard, von der Grün se retrouve dans un camp de prisonniers aux Etats-Unis, duquel il ne rentre en Allemagne qu'en 1948. Tout ce que l'on lit sur ses années de l'après-guerre est que les conditions matérielles dans lesquelles il les a vécues étaient meilleures pour lui que pour la majorité des Allemands. Les réflexions qui découlent des documents sur les crimes nazis révélés au public après la fin de la guerre ne portent pas sur les réactions directes de la population, mais sur l'oubli dans lequel les crimes contre l'humanité semblent risquer de tomber au moment où l'auteur écrit son livre.

20 Aussi bien Günter de Bruyn que Dieter Wellershoff accordent plus de place à ce qu'ils ont vécu dans l'après-guerre. Mais le combat pour la patrie auquel ils ne croyaient plus depuis quelque temps (contrairement à Ludwig Harig qui confesse honteusement avoir écrit des poèmes à la plus grande gloire des nazis jusqu'en avril 45) se transforme dans 
un premier temps sur le plan des événements relatés en un combat solitaire pour la survie pour céder ensuite la place à une narration centrée presque exclusivement autour de la vie privée, lorsque celle-ci retrouve un cours plus paisible. Rares sont les allusions à la reprise hésitante de la vie politique et c'est au lecteur d'en voir éventuellement une quand Wellershoff dit à propos des fêtes familiales de la fin des années 40 auxquelles il a assisté qu'elles se déroulèrent dans une "ambiance d'irresponsabilité sereine $»^{23}$.

Dans Zwischenbilanz, l'arrière-fond extérieur reste arrière-fond à un point que l'on ne se rendrait presque pas compte que l'action se déroule sur le territoire de ce qui était en train de devenir la RDA si elle n'était pas de temps à autre située géographiquement. Seul un questionnaire que de Bruyn doit remplir avant d'intégrer l'équivalent de l'Ecole Normale - du reste la seule «mesure » de dénazification évoquée dans le livre - semble clairement inspiré de l'idéologie des forces d'occupation soviétiques. Est-ce parce que de Bruyn déclare avoir le projet d'un deuxième livre autobiographique, consacré celuici a ses quarante ans de vie en RDA qu'il ne saisit pas l'occasion de refléter son rôle comme citoyen dans ce système politique qui commence à s'installer ? La question sur l'absence d'interrogations de ce type pourrait également s'adresser à Wellershoff à qui on ne peut pourtant pas reprocher d'avoir voulu peindre un tableau apologétique ou édulcoré de son temps passé au "régiment auxiliaire Hermann Göring ». Ce reproche ne pouvant véritablement être fait à aucun des quatre auteurs, il n'en reste pas moins que la césure dans les priorités des récits autobiographiques ressort nettement.

On pourrait voir dans cet état de chose une confirmation de la théorie quelque peu extravagante de Bernd Neumann $^{24}$ selon laquelle la possibilité d'écriture autobiographique disparaît avec la fin de l'adolescence, une fois que l'individu a adopté son rôle à jouer dans la société. Dans ce cas, il serait possible de préciser que pour les auteurs n'ayant pas eu l'occasion de choisir ce rôle dans la relative liberté dont auraient disposé d'autres générations, la tâche d'écrire des textes autobiographiques qu'ils se sont donnée correspond simultanément à un besoin ou même à un devoir de témoigner et à une manière de chercher quels ont été les facteurs qui ont déterminé leur évolution et qui ont laissé si peu de place à des alternatives. Mais c'est justement cet aspect de la question qui peut aussi venir infirmer la théorie citée ci-dessus: les écrivains de la génération concernée n'ont pas pu opter de leur propre chef pour un rôle avant d'entrer dans la vie d'adulte : les textes ne constitueraient donc pas le récit d'une recherche, mais ils seraient eux-mêmes cette recherche qui n'a pas pu se dérouler « normalement ».

Ce sont les conditions dans lesquelles sont devenus adultes ces auteurs à qui on avait volé leur jeunesse, qui proposent une éventuelle piste pour expliquer la prédilection manifeste dans le choix de la période à élucider : l'opacité sur le plan de la psychologie individuelle qui est propre à leur jeunesse et qui réclame donc quasiment que l'on s'interroge sur elle, contraste avec la clarté apparente avec laquelle on peut fixer le début et la fin de la période correspondante sur le fil de l'histoire allemande. Plus une étape reste énigmatique, plus la volonté se fait sentir de soulever des questions à son sujet, mais plus le besoin semble grand aussi de se repérer à des facteurs supraindividuels qui permettent de mieux la saisir. En ce qui concerne l'empire douzenaire, ces repères sautent extérieurement aux yeux.

Reste à invoquer dans ce même contexte que la période de 1933 à 1945 a beaucoup plus souvent fait l'objet de recherches que celle de l'après-guerre. Il existe donc une 
littérature abondante qui a pu permettre aux auteurs respectifs de refléter leur passé à la lumière d'informations utiles pour accéder au niveau d'abstraction, au degré d'objectivité indispensables pour pouvoir placer le moi comme sujet au centre de la création littéraire. l'époque qui va jusqu'en 1945 pour ensuite présenter ce qu'il est devenu dans l'aprèsguerre. Evidemment, Rühmkorf aussi part de son point de vue personnel, il évoque donc principalement l'évolution des zones d'occupation occidentales ainsi que celle de la RFA à travers les répercussions qu'il en a ressenties dans sa carrière de journaliste et d'auteur lyrique. Mais le point de vue personnel de l'auteur politiquement engagé qu'il est n'est pas celui d'une personne privée ; la littérarité autobiographique coïncide avec l'acception que de Bruyn avait proposée et réside ici justement dans l'incorruptibilité du regard qu'il porte aussi bien sur la vie littéraire et journalistique renaissante - la liberté de la presse est inscrite sur du papier qui appartient à un groupe de gens bien particulier ${ }^{26}$ - que sur son propre rôle d'acteur de cette vie littéraire. Car ce n'est pas seulement son jugement sur «Mesdames et Messieurs les ignorants, il faudrait vous couper en quatre, ou au moins le pays dont vous êtes issus $»^{27}$, mais aussi sur ses propres activités qu'il qualifie dans leur inefficacité de "poursuite de l'idéalisme allemand avec d'autres moyens $\aleph^{28}$ : ce jugement est tout aussi dur et cru que par exemple celui de Wellershoff sur le nazisme.

Il est donc possible d'écrire sur sa vie en Allemagne dans une continuité, mais notre comparaison invite à penser qu'il y a pour cela une concession à faire, même si la justesse de cette supposition reste à vérifier à travers un travail plus large. La continuité que nous avons implicitement postulée semble d'un côté relativisée par la contrainte selon laquelle il faut avoir oublié avant de pouvoir se souvenir. Il s'agit par conséquent d'un passage obligé précédant l'écriture d'un texte autobiographique au sens étroit du terme. C'est cette qualification que nous donnons malgré une certaine diversité formelle aux récits des quatre premiers auteurs qui ont fait l'objet de la 
présente analyse. Ce qui oppose leurs textes à celui de Rühmkorf est le fait que ce dernier, de son côté, a dû faire la concession esthétique de rapprocher son livre du journal afin d'avoir un regard sur sa vie qui ne soit pas sélectif dans le temps.

Comme piste de recherche se proposeraient de plus amples interrogations sur d'une part les causes de la négligence de l'immédiat après-guerre chez les autobiographes, question dont nous n'avons pu présenter ici qu'une ébauche, ainsi que d'autre part un questionnement sur les possibilités narratives que le journal factice semble offrir.

\section{NOTES}

1. - Wellershoff, Dieter, Die Arbeit des Lebens (ADL), Cologne, 1985.

2. - Wellershoff, Dieter, Der Ernstfall, Cologne, 1995.

3. - de Bruyn, Günter, Zwischenbilanz - Eine Jugend in Berlin (ZB), Francfort, 1992.

4. - Von der Grün, Max, Wie war das eigenlich? - Kindheit und Jugend im Dritten Reich (WWDE), Hamburg, 1979.

5. - Harig, Ludwig, Weh dem, der aus der Reihe tanzt (WDRT), Munich, 1990.

6. - Rühmkorf, Peter, Die Jahre, die Ihr kennt - Anfälle und Erinnerungen (DJIK), Hambourg, 1972.

7. - ADL, p. 14.

8. - Cf., Fühner, Ruth, Das Ich im Prozess, Fribourg, 1982, p. 23.

9. - Cf., ADL, p. 45.

10. - Cf., $A D L$, p. 48.

11. - Cf., $A D L$, p. 51.

12. - ADL, p. 72 : «Das kollektive Drama war in viele Millionen individuelle Dramen zerfallen, [...]» 13. - De Bruyn, Günter, Das erzählte Ich - Über Wahrheit und Dichtung in der Autobiographie (DEI), Francfort-sur-le-Main 1995. Pour le passage suivant : cf. surtout pp. 33 sqq.

14. - ZB, p. 22/23 : «[...] der Tag meiner Geburt [...] an der Front.»

15. - Op. cit., cf. p. 53.

16. - DEI, p. 74 : «Es passt aber gut.»

17. $-Z B$, p. $2:$ «[...] Entwicklungsroman und Epochenpanorama in einem [...]».

18. - Cf., WWDE, p. 2.

19. - Cf., WDRT, p. 239.

20. - Cf., WDRT, p. 226.

21. - WDRT, p. 272 : «[...] ich kann nichts ungeschehen machen.»

22. - WWDE, p. 226.

23. - ADL, p. $102:$ «[...] in heiterer Verantwortungslosigkeit.»

24. - Cf., Neumann, Bernd, Identität und Rollenspiel, Francfort 1970, pp. 11/12 et 22.

25. - Cf., DEI, p. 48.

26. - DJIK, p. 41 : «Die Pressefreiheit steht auf Papier, das ganz bestimmten Leuten gehört.»

27. - DJIK, p. 19/20 : «[...] meine Damen und Herren Nixwisser [...] vierteilen sollte man euch, oder zumindest dieses Land, das euch hervorgebracht hat.»

28. - DJIK, p. 53 : «Fortsetzung des deutschen Idealismus mit anderen Mitteln». 


\section{RÉSUMÉS}

L'objet de cet article est de montrer avec quels moyens narratifs cinq auteurs contemporains, c'est-à-dire Dieter Wellershoff, Günter de Bruyn, Max von der Grün, Ludwig Harig et Peter Rühmkorf se penchent dans leur autobiographie sur l'époque nazie et celle de l'après-guerre. Quel poids respectif accordent-ils aux deux périodes? Il s'agit dans un premier temps d'exposer les différentes approches dont se servent ces écrivains pour porter un regard sur leur jeunesse sous le Troisième Reich et de définir la place que cette époque occupe dans le texte autobiographique; on s'interroge ensuite sur les éléments et les critères narratifs qui changent chez les uns et les autres et sur le mode selon lequel ce changement s'opère dès que c'est l'après 45 qui est évoqué. Il faut finalement se demander dans quelle mesure le postulat narratologique de la continuité exige, de la part de l'autobiographe, des concessions esthétiques et formelles qui le rapprochent d'un auteur qui écrirait son journal « a posteriori ».

Gezeigt werden soll, mit welchen erzählerischen Mitteln und mit welcher Gewichtung fünf zeitgenössische Autoren, nämlich Dieter Wellershoff, Günter de Bruyn, Max von der Grün, Ludwig Harig und Peter Rühmkorf in ihren Autobiographien den Nationalsozialismus und die Nachkriegsjahre beleuchten. Es geht zunächst darum, die verschiedenen Ansätze darzulegen, derer sich die Schriftsteller bedienen, um auf ihre Jugend im Dritten Reich zu blicken und den Raum zu umreissen, welchen diese Epoche im autobiographischen Text einzunehmen scheint, um anschliessend darauf einzugehen, wie und bei wem sich welche Textmerkmale verändern, sobald die Zeit nach 1945 zum Gegenstand gemacht wird. Schliesslich muss sich gefragt werden, inwiefern das narrative Postulat der Kontinuität vom Autobiographen ästhetische und formale Zugeständnisse verlangt, die ihn in die Nähe eines «nachträglichen» Tagebuchschreibers rücken.

\section{AUTEUR}

\section{KERSTIN ADAM}

Université Charles-de-Gaulle - Lille III 